\title{
Electrophysiological Properties of Cochlear Implantation in the Gerbil Using a Flexible Array
}

\author{
Christine DeMason, Baishakhi Choudhury, Faisal Ahmad, Douglas C. Fitzpatrick, Jacob \\ Wang, Craig A. Buchman, and Oliver F. Adunka \\ Department of Otolaryngology-Head and Neck Surgery, University of North Carolina at Chapel \\ Hill.
}

\begin{abstract}
Objectives-Cochlear implants (CI) perform especially well if residual acoustic hearing is retained and combined with the $\mathrm{CI}$ in the same ear (also termed hybrid or electric-acoustic stimulation). However, in most CI patients, residual hearing is at least partially compromised during surgery, and in some it is lost completely. At present, clinicians have no feedback on the functional status of the cochlea during electrode insertion. Development of an intraoperative physiological recording algorithm during electrode insertion could serve to detect reversible cochlear trauma and optimal placement relative to surviving hair cells. In this report, an animal model was used to assist in determining physiological markers for these conditions using a flexible electrode similar to human surgery.
\end{abstract}

Design-The animal model was the normal-hearing gerbil. The flexible electrodes had 1 to 2 platinum-iridium contacts embedded in a $200 \mu \mathrm{m}$ diameter silastic carrier. As control experiments some insertions were also made with much smaller ( $50 \mu \mathrm{m}$ diameter) rigid electrodes. In either case, the electrode was positioned at or just inside the round window membrane and subsequently advanced into the scala tympani longitudinally in 50 to $100 \mu \mathrm{m}$ increments. After each advancement, acoustic stimulation was used to elicit a cochlear microphonic (CM) and compound action potential (CAP). Stimuli were suprathreshold tone bursts of 1 to $16 \mathrm{kHz}$ in octave steps with $2 \mathrm{msec}$ rise and fall times and a $10 \mathrm{msec}$ plateau. Anatomical integrity of the cochlea was subsequently assessed using a whole-mount preparation.

Results-In contrast with the CAP, which was relatively stable during insertion, the CM showed a variety of changes related to electrode movement. To tone bursts of 1 to $8 \mathrm{kHz}$ the $\mathrm{CM}$ typically remained stable or increased during the insertion before contact with cochlear structures. After contact, the potentials often dropped dramatically. The $\mathrm{CM}$ to $16 \mathrm{kHz}$ was the most variable; in some cases it increased but in other cases it decreased early in the insertion and later showed large and abrupt increases. In some instances, this pattern was seen to progressively lower frequencies as well. Histological analysis and the gerbil frequency map indicate that electrode travel was limited to the basal turn ( $4 \mathrm{~mm}$ from the hook) and did not intrude into the characteristic frequency regions of most frequencies used.

Conclusions-First, the CM provides a more sensitive indication of cochlear trauma than does the CAP. Second, stable or steady increases in the CM are a physiological marker for unimpeded travel through the scala tympani as the electrode approaches responding hair cells. Third, abrupt reductions in the CM across frequency are a physiological marker of contact with cochlear structures. Fourth, abrupt increases after a decline, which occurred primarily to $16 \mathrm{kHz}$ but to a

Copyright $(92012$ by Lippincott Williams \& Wilkins

Address for correspondence: Oliver F. Adunka, MD, University of North Carolina at Chapel Hill, Chapel Hill, NC, USA. oadunka@mac.com. 
lesser degree to other frequencies as well, are a physiological marker for a release from contact. The interpretation is that as the tip of the electrode bends the shaft can move in the mediolateral dimension, sometimes contacting the basilar membrane and sometimes not. Overall, the results indicate that recordings during cochlear implantations can provide valuable feedback to the surgeon regarding electrode position and the integrity of surviving hair cells.

\section{INTRODUCTION}

Patients with some residual hearing are increasingly receiving cochlear implants (CI) to improve their ability to communicate using speech (Cullen et al. 2004). In these individuals, the introduction of an electrode can improve speech understanding as in conventional implantations, and if hearing is preserved, a benefit especially in the presence of background noise can be achieved (Gstoettner et al. 2008; Buchner et al. 2009; Gantz et al. 2009). Because of the benefits of preserving residual hearing, several clinical protocols have been established, including electric-acoustic stimulation (EAS), "hybrid" cochlear implantation, and partial deafness surgery (von Ilberg et al. 1999; Skarzynski et al. 2006; PodskarbiFayette et al. 2010; Turner et al. 2010).

The most common approach to preserve residual hearing is to use a shallow insertion. Very short electrodes $(6 \mathrm{~mm})$ have been used to minimize trauma, resulting in a high success rate for hearing preservation (HP) surgery (Gantz et al. 2005; Turner et al. 2010). However, in cases where residual hearing is poorly preserved or is progressive, a very short electrode configuration is suboptimal. With the advent by CI manufacturers of smaller, softer, and more flexible electrode technology insertions up to one full turn $(20-25 \mathrm{~mm})$ are in current use (Adunka et al. 2004; Gstoettner et al. 2004; Fraysse et al. 2006; Helbig et al. 2011). Still, typical insertions in profoundly deaf patients are up to $30 \mathrm{~mm}$, and deep insertions are considered beneficial to reach the low-frequency portion of the cochlear tonotopy (Hochmair et al. 2003). In addition, in many implantations of deaf individuals a semirigid, precoiled electrode is used to hug the modiolus and be in close proximity to spiral ganglion cells (Eshraghi et al. 2003; Adunka et al. 2006). A flexible, free-fitting electrode that occupies a position lateral in the scala tympani is an acceptable compromise to preserve hearing, but might be less optimal when considering electric hearing alone (Holden et al. 2011). For these reasons it would be beneficial to know, intraoperatively, the likelihood that hearing has been compromised. Such physiological information could be used to guide the surgeon in determining whether to optimize for EAS or electrical stimulation alone. The recording could also potentially alert the surgeon that further travel on a given path would likely be traumatic, or that the electrode had reached an optimal position relative to surviving hair cells.

A gerbil model has recently been developed to identify electrophysiological markers of cochlear health during a cochlear implantation where preservation of residual hearing is the goal (Adunka et al. 2006, 2010; Campbell et al. 2010a, 2010b; Choudhury et al. 2011; Subermanet al. 2011). We have shown that a range from very small to very large degrees of damage to hair cells can be detected from intracochlear responses to tones. The degree of physiological reduction of response correlates with the magnitude of damage observed anatomically, from small disruptions of a few hair cells to large holes in the basilar membrane (BM) or the osseous spiral lamina (OSL). Small response decrements are reversible upon electrode retraction. Intense stimuli are used so that the potentials are large with little averaging required, and small changes in response can be detected over wide regions with a single frequency/intensity combination. Therefore, the speed and precision of detecting electrode contact is high. The conclusion from these experiments is that physiological indications of hair cell integrity can be available on-line to guide the surgeon about decisions based on the preservation or loss of residual hearing. In this report, the 
model is extended to more fully characterize insertion effects on intracochlear physiology. The previous experiments used a rigid electrode with the electrode oriented radially, that is, toward the BM. This experimental design was chosen to correlate physiological changes with anatomical contact and degree of damage. However, a soft, flexible electrode oriented in the same direction as the BM is more analogous to a human surgery. Consequently, the experiments described here use flexible electrodes, and the insertions are oriented longitudinally rather than radially to better reflect real human cochlear implantation. To test the degree to which results were attributable to the flexibility of the electrode used, we also used a rigid electrode for additional experiments with longitudinal insertions. The main aim of this work is to find a reliable signal for knowing when insertion of the cochlear electrode causes damage during implantation.

\section{MATERIALS AND METHODS}

\section{Animals}

The Mongolian gerbil (Meriones unguiculatus) was used because its low-frequency hearing range is similar to humans and its cochlea is easily accessible through the large auditory bulla. All animals were handled and housed according to the standards described by the National Institutes of Health Committee on Care and Use of Laboratory Animals, using protocols approved by the Institutional Animal Care and Use Committee at the study institution.

\section{Animal Handling}

Surgeries and recordings were performed under deep urethane anesthesia (25\% solution in saline, $1.5 \mathrm{~g} / \mathrm{kg}$, i.p.). Once anesthesia was induced, the animal was moved to a doublewalled, sound-attenuated booth. The core body temperature was monitored with a rectal probe and maintained around $37^{\circ} \mathrm{C}$ with heating pads.

\section{Surgical Procedure}

The bony bulla was exposed and opened via a standard postauricular skin incision. The pinna was removed to allow for consistent sound delivery. The round window niche, the stapedial artery, and all other structures of the bulla were identified. A small slit was made in the round window membrane and an intracochlear recording electrode was positioned at or just inside (up to a few hundred micrometer) the membrane using a micromanipulator.

\section{Acoustic Stimulation and Calibration}

For most cases (17/21) the stimuli were tone bursts of 1 to $16 \mathrm{kHz}$ in octave steps at either $60 \mathrm{~dB}$ SPL or at varying dB SPL for all responses to be similar to the $4 \mathrm{kHz}$ at $60 \mathrm{~dB}$ SPL response. The bursts had a $10 \mathrm{msec}$ plateau and $2 \mathrm{msec}$ rise/fall times and a $30 \mathrm{msec}$ interstimulus interval. For some cases $(4 / 21)$ only a single frequency $(4 \mathrm{kHz})$ at $60 \mathrm{~dB}$ SPL was used. To deliver the stimuli, the electrical signals were generated and delivered to a well-shielded loudspeaker (Beyer DT-48; Farmingdale, NY) using custom software, a National Instruments input/output board (model 6250E; Austin, TX), and a Tucker-Davis system III headphone buffer (model HB7; Alachua, FL). The speaker was $10 \mathrm{~cm}$ from the animal's tympanic membrane. Calibration was performed using a 1/4" Bruel \& Kjaar (Nærum, Denmark) microphone placed at the position of the animal's eardrum, and levels were presented in $\mathrm{dB}$ SPL (re $20 \mu \mathrm{Pa}$ ).

\section{Electrode and Recording Configuration}

Two different electrodes were used in this experiment. One was a flexible electrode supplied by the MED-EL Corporation (Innsbruck, Austria) that had one to two iridium contacts 
embedded in a $200 \mu \mathrm{m}$ diameter silastic carrier. The other electrode was a $50 \mu \mathrm{m}$ diameter, Teflon-insulated tungsten iridium rigid wire with approximately $50 \mu \mathrm{m}$ of tip insulation removed. The electrodes were attached to a hydraulic micromanipulator that was controlled from outside the sound booth and advanced in steps of 50 to $100 \mu \mathrm{m}$. The recording was differential and monopolar, with the electrode connected to the positive lead of the amplifier (Grass Instruments model P15D, West Warwick, RI), the return electrode to the neck musculature, and the ground to the tail. Gain was $1000 \times$ and filters were band pass from 10 to $50,000 \mathrm{~Hz}$. The amplifier signal was digitized ( $200 \mathrm{kHz}$ sampling rate), and 20 stimulus repetitions were averaged at each frequency. Three blocks of the 20 averages were obtained for each electrode location.

\section{Intracochlear Recordings and Data Analysis}

The insertions were longitudinal in the scala tympani. The initial recordings at or just inside the round window (RW) were the baseline used for comparison with all subsequent recordings. A fast-Fourier transform (FFT) was used to estimate the cochlear microphonic (CM) magnitude at the signal frequency, after filtering (0.5-2 octaves around the stimulus frequency) and windowing $(6-11 \mathrm{msec})$ to avoid the compound action potential (CAP). The magnitude of the CAP was taken as the root mean square value of the epoch from 1 to 6 msec after filtering $(0.5-1.5 \mathrm{kHz})$. The experiment consisted of advancing an electrode through the basal turn and detecting changes in the CM and the CAP as the electrode impacted the BM/OSL complex. At each location the mean and SD of the three blocks was calculated. When response changes were detected, the electrode could be withdrawn to determine whether the effect was reversible, indicative of an effect without lasting damage. When sharp declines were detected in most of the frequencies that did not reverse with retraction of the electrode, recordings were taken in increments of $15 \mathrm{~min}$ to test for reversibility with time. The flexible electrode was in some cases manually manipulated if the tip was bent or blocked on any cochlea structures.

\section{Assessment of Cochlear Status}

Each animal was sacrificed, and the cochlea was removed en block. The whole mounts were decalcified, bone was removed for better visualization of cochlear structures, and the block was stained with toluidine blue. Cochlear damage caused by the electrode was photographed at 10 to 50x using a Wild M50 dissecting microscope (Leica Inc., Wetzlar, Germany). Distance to the damage was measured from the basal end of the BM in the hook region (see arrow in Fig. 5). The distance reported for the recordings is from the RW, which was 1.4 $\mathrm{mm}$ from the hook on average.

\section{RESULTS}

\section{Results With Flexible Electrode Insertions}

A variety of frequency-specific response changes during insertion were observed. An illustrative case is shown in Figures 1 and 2. The response to tones of different frequency at the beginning of the insertion (Fig. 1) served as a reference during the rest of the procedure. Consequently, the magnitude of each curve was normalized (see Fig. 1 legend for raw values). The waveforms (Fig. 1A) were composed of both the CM and the CAP. The CM magnitude (Fig. 1B) was determined from a fast-Fourier transform within a window that excluded the CAP (Fig. 1A, box). The CAP magnitude was measured from a filtered version of the raw waveform (Fig. 1C).

Changes in the magnitudes of the $\mathrm{CM}$ and the $\mathrm{CAP}$ at a single intensity were measured as the electrode was inserted (Fig. 2A). The data are plotted as a function of trial number with some time points of the experiment shown below. The top panel represents the depth of the 
insertion relative to the RW. Step sizes were $50 \mu \mathrm{m}$ up to trial $21(869 \mu \mathrm{m})$. At each frequency (bottom five panels), the CM showed relatively large changes whereas the CAP was more stable. The responses increased smoothly as the insertion began, consistent with longitudinal or mediolateral movements toward responding hair cells and nerve fibers while the electrode traversed the scala tympani nontraumatically. There was a sharp change in the $\mathrm{CM}$ to all frequencies at trial 20 (arrows, $869 \mu \mathrm{m}$ ). Before this change, there was some variability across frequencies: to 4 and $8 \mathrm{kHz}$ the $\mathrm{CM}$ continuously increased, to 1 and 2 $\mathrm{kHz}$ it had reached a plateau and begun to decline, and to $16 \mathrm{kHz}$ it had declined considerably. The change at trial 20 was not the same across frequencies; the CM declined to 1 and $8 \mathrm{kHz}$ but increased to $16 \mathrm{kHz}$.

In this case, the electrode was fixed in place with wax and the end outside the cochlea was cut. The cochlea was then prepared as a whole mount (Fig. 2B). This procedure could not be routinely done as the electrodes were used for multiple experiments, but it provides useful visual orientation of the relative size of the electrode and scala tympani in the gerbil's basal turn.

Another example that further illustrates the complex pattern of results during an insertion is shown in Figure 3. As with the previous example, the CM to each frequency showed relatively large changes whereas the CAP was more stable. However, at the end of the experiment when irreversible damage occurred, the CAP decreased along with the $\mathrm{CM}$. To describe the complex pattern of changes in the CM, different inflection points are indicated by numbers. At point 1 (trial 6, depth $0.22 \mathrm{~mm}$ ), the $\mathrm{CM}$ to all frequencies was increasing, except to $1 \mathrm{kHz}$ where it was stable until nearly the end of the insertion. The increased CM continued to 2 and $8 \mathrm{kHz}$, but after point 1 the response to $16 \mathrm{kHz}$ began a gradual decline. At point 2 (trial 18, depth $0.83 \mathrm{~mm}$ ), the $\mathrm{CM}$ to 2 and $8 \mathrm{kHz}$ showed a sharp decrease that was largest to $8 \mathrm{kHz}$, whereas the $\mathrm{CM}$ to $16 \mathrm{kHz}$ sharply increased. At this point the electrode was partially withdrawn, and all of the changes reversed. Between points 2 and 3, there was another increase to $16 \mathrm{kHz}$ (trial 26). After further insertions, the response to 16 $\mathrm{kHz}$ returned to its previous low level. At point 3 (trial 38, depth $1.37 \mathrm{~mm}$ ) the $\mathrm{CM}$ to 2 and $8 \mathrm{kHz}$ showed a sharp decline but that to $16 \mathrm{kHz}$ did not rebound. With further insertion the $\mathrm{CM}$ to $8 \mathrm{kHz}$ declined further and that to $4 \mathrm{kHz}$ began a gradual decline. At point 4 (trial 54, depth $2.02 \mathrm{~mm}$ ) the $\mathrm{CM}$ to $2 \mathrm{kHz}$ showed a small but abrupt decline, whereas the CMs to 4 and $8 \mathrm{kHz}$ increased (the increase to $8 \mathrm{kHz}$ was small but apparent using a different scale). At point 5 (trial 59, depth $2.22 \mathrm{~mm}$ ) large declines began to all frequencies that did not reverse when the electrode was withdrawn.

After the experiment, a $0.39 \mathrm{~mm}$ hole in the BM was present (Fig. 3B). This hole was 1.31 to $1.70 \mathrm{~mm}$ from the RW (2.58-2.97 $\mathrm{mm}$ from the hook). This hole was not as deep as the insertion depth, suggesting that the damage was caused by the shaft of the electrode rather than the tip. There was also bruising on the OSL near the RW where the electrode scraped during insertion.

The prevalence of the main points described in the examples is shown in Table 1.

Anatomical damage caused by the electrode was seen in 7 of the 21 cases, no damage could be identified in 11 cases and in 3 cases histology was not available because of issues during specimen processing. Even in cases with damage, only four had a clear hole in the BM; the others had disruptions of support cells indicating that the BM and the organ of Corti were stressed at that point. Fifteen of the 21 cases showed that the $16 \mathrm{kHz}$ response decreased with advancement, sometimes followed by a progressive decrease at other frequencies, as in the case in Figure 3. In one case there was no clear decline even to $16 \mathrm{kHz}$ until near the maximum depth. Reversibility when the electrode was withdrawn early in the insertion was seen in 13 cases. In the remaining eight cases depth was progressively increased, and 
reversibility was not tested. Irreversible damage was seen at the end of the insertion in 10 cases, often after manual manipulation once the electrode was seen to be bending with the microscope. In three cases all of the response changes reversed when the electrode was withdrawn, and in seven cases this type of reversibility was not tested.

\section{Results With Rigid Electrode Insertions}

One of the results from the insertion with flexible electrodes was that the CM initially increased but then declined in a progressive manner from high to low frequencies. This pattern is illustrated in Figure 3, where the CM to $16 \mathrm{kHz}$ declined first, followed in order by the other frequencies. We think this pattern indicates contact of the electrode over an increasing extent of the BM, causing progressive loss of contributions to each frequency. If so, this pattern should be less evident or absent by simple progression through the scala tympani without contact with cochlear structures. To test this, a series of longitudinal insertions was made with a much smaller electrode $(50 \mu \mathrm{m}$ diameter rigid wire versus 200 $\mu \mathrm{m}$ diameter silastic carrier).

An example of a rigid electrode insertion is shown in Figure 4A. As with the examples with flexible electrodes, the $\mathrm{CM}$ to each frequency showed relatively large changes, whereas the $\mathrm{CAP}$ was more stable. Until trial 12, the CMs to 4 and $16 \mathrm{kHz}$ rose continuously, while the CMs to 1 and $2 \mathrm{kHz}$ were stable. At trial 12 all frequencies showed a sharp decline. The $\mathrm{CAP}$ to 1 and $2 \mathrm{kHz}$ also dropped. None of the responses recovered with small retractions of the electrode or, after trial 15, when electrode was withdrawn to the RW. The cochlea had a hole in the BM (Fig. 4B) 1.14 to $1.39 \mathrm{~mm}$ from the RW (2.56-2.81 mm from the hook).

This case is representative because of the 11 insertions made with rigid electrodes, 7 showed no evidence of frequency-specific loss of response and none showed sharp increases in the $\mathrm{CM}$ to $16 \mathrm{kHz}$ or any other frequency (Table 2).

\section{Anatomical Considerations}

The locations of damage caused by our insertions are shown in Figure 5A. To make as much of the cochlea in focus as possible, this picture is a composite of several frames, each taken with a different focus and then superimposed to make a montage in Photoshop. The depths of each site can be obtained from Tables 1 and 2 on the basis of the gerbil number indicated (depths in Tables 1 and 2 are from the end of the hook, indicated by the arrow in Fig. 5A). The deepest evidence of contact was from insertions using the flexible electrodes and extended to about $10 \mathrm{kHz}$ characteristic frequency (CF) of cochlea 9. The damage seen with rigid electrodes overlapped that made with flexible electrodes, but in general did not extend as far (maximum $\sim 3 \mathrm{~mm}$ ). Using rigid electrodes, the damage typically correlated well with the maximal depth of insertion. In contrast, the damage from flexible electrodes was typically shallower than the deepest depth of insertion, although in the cases of deepest damage (most apical red dots) the correspondence was good. As in the example in Figure 3, we interpret these results as evidence that with the flexible electrodes damage could be caused by the shaft rather than tip of the electrode.

The deepest insertions with the flexible electrode were about $4.1 \mathrm{~mm}$ from the basal end of the cochlea or about $2.7 \mathrm{~mm}$ from the RW. This region corresponds to where the scala tympani narrows and prevents further advance of the electrode (Fig. 5B, arrow).

\section{DISCUSSION}

Response changes because of electrode travel were complex. The physiological changes were consistent with a mode of travel where there was no contact with cochlear structures or a mode where there was contact and consequent reduction of response. In the following 
section, we will first discuss the animal model used and then consider the sources of the changes in potentials observed during insertions.

\section{The Normal-Hearing Model}

The normal-hearing gerbil model has several benefits and some weaknesses. For measurement purposes, the good hearing status provides a sensitive measure of cochlear health that can be correlated with anatomy. The source and nature of the potentials allow for particular interpretations of electrode position, as we will describe in the subsequent paragraphs. As for the hearing condition itself, it is not typical of the basal turn in an HP surgery, which would be devoid of hair cells. Thus, the penetrations reported here most closely simulate the condition where an electrode has already encountered surviving hair cells. The results indicate that if the electrode is small relative to the scala tympani it can traverse within regions of intact hair cells with little effect on response. The demonstration of reversible response losses shows that damage can potentially be detected in time to avoid irreversible trauma. In many cases, the flexible electrode did not cause visually observable damage, and when it did, it rarely pushed into other compartments, indicating that a very soft electrode can be mechanically nontraumatic. A natural extension of this work would be to perform similar experiments using the recently developed gerbil model with noiseinduced hearing loss that better simulates the anatomical conditions in the basal turn of a typical HP patient (Choudhury et al. 2011; Suberman et al. 2011).

All cases with obvious anatomical damage in the whole-mount preparations as a result of electrode contact showed irreversible changes in response after the electrode was withdrawn from its deepest insertion (Tables 1 and 2, late reversibility). Three cases with the flexible electrode and one case with the rigid electrode did not show obvious anatomical damage but did show irreversible changes. Each of these cases showed some displacement of the BM or cells in the organ of Corti that was likely to be caused by electrode contact. However, it was not uncommon for similarly small displacements to occur during the dissection process. If the damage was not greater than what could have been caused by handling we did consider it to have been clearly caused by the electrode.

\section{Physiological Markers of Trauma-Free Insertion}

In the early parts of insertions with either a flexible or a rigid electrode the responses often rose at all frequencies. We interpret this rise as indicating nontraumatic travel through the scala tympani. In the normal-hearing animal, the rise could be attributable to longitudinal movement toward the $\mathrm{CF}$ region of the stimulating frequency, but radially directed insertions also show this increase (Campbell et al. 2010a, 2010b), suggesting that at suprathreshold intensities source generators are located in the basal turn itself and movement toward the BM will get closer to them. In an HP surgery it is likely that the potential would rise at some point as intact elements were approached, but the degree to which the rise would be detectable in the basal turn is not yet known.

\section{Physiological Markers of Electrode Contact and Damage}

Changes associated with light contact to the BM were mainly detectable as reductions in the $\mathrm{CM}$ at higher frequencies. Reductions in the CAP across frequency or to the $\mathrm{CM}$ at low frequencies were associated with more severe contact and damage. These results are explicable based on the different sources of the CM and the CAP, which will be described in turn.

The CM is linear with intensity with saturation occurring only at the highest intensities (> 85 dB SPL) (Adunka et al. 2010; Campbell et al. 2010a). At threshold, the region slightly above the CF provides the greatest contribution to the CM, because the contribution 
at the CF region largely cancels as a result of rapid phase changes (Cheatham et al. 2011). As the intensity increases, more basal and apical hair cells will contribute to the response as the slope of the phase change for frequencies near CF declines. At high intensities the contributing region will spread basally but because of the linear input-output response the contribution from $\mathrm{CF}$ and near $\mathrm{CF}$ regions increase as well. Thus, the center of gravity of the source of the CM will shift basally but with a strong "pull" from the CF region. This means that at the highly suprathreshold intensities used in this report there should be a contribution from basal hair cells that scales as a function of frequency, which is the essential result observed. The progression of frequency loss was not consistent with a tonotopic passage of CF regions, because of the following reasons: (1) The same pattern was in general not seen with the smaller, more rigid electrode that traversed the same space; (2) The recording was monopolar, so there was no differential sampling to localize responses to a particular CF region (Schmiedt \& Zwislocki 1977); and (3) the CF region of even the highest frequency $(16 \mathrm{kHz})$, was not passed until the insertion was deep and beyond where reductions in the $\mathrm{CM}$ to this frequency were usually noted. The identification of sharp increases as well as decreases in the $\mathrm{CM}$ to $16 \mathrm{kHz}$ and then progressively at lower frequencies is also consistent with the interpretation of basal suppression rather than tonotopy. We interpret these increases as attributable to a release of contact as the electrode bends differentially at the tip and shaft. The overall preservation of response at $1 \mathrm{kHz}$ compared with the other frequencies is also consistent with the interpretation of a generator region close to its $\mathrm{CF}$ and relatively remote from the basal turn compared with the other frequencies. This result is also suggested using the noise-induced hearing loss model, where responses to $1 \mathrm{kHz}$ are only slightly reduced compared with normal-hearing animals (Choudhury et al. 2011; Suberman et al. 2011).

In contrast with the CM, growth functions of the CAP are highly saturating (Adunka et al. 2010; Campbell et al. 2010a). Saturation arises because the source of the CAP is the synchronized initial action potentials of auditory nerve fibers to the onset of a stimulus. Because the onset response is limited to one or a few spikes, in any given fiber it is nearly the same at all intensities above threshold. Consequently, basal spread will be rapid with intensity, and there will be little pull from the CF region. At the high intensities used here the CAP is well in the saturated range. Because a reduction in the magnitude of a potential is analogous to a decrease in intensity, the saturation of the CAP can explain its relative stability compared with the CM. Loss of input over a greater proportion of the cochlea should be needed before a reduction in the CAP would be expected.

\section{Indications for HP Surgery}

Electrode travel in the gerbil was limited to the basal turn where the scala tympani is large. The diameter of the flexible electrode was $200 \mu \mathrm{m}$, and beyond the basal turn the scala tympani diameter is slightly smaller than this. In humans, at regions where intact hair cells might be expected in an HP surgery ( $>50 \%$ of the distance to the apex) the scala tympani in the human narrows to about $1 \mathrm{~mm}$. Thus, an electrode could be $\sim 300 \mu \mathrm{m}$ in diameter and have the same relative dimensions as the electrode used here in the gerbil basal turn. Current electrodes used for an HP cochlear implantation are about 0.5 to $0.8 \mathrm{~mm}$ in diameter and hence are relatively larger, but are still smaller than the cochlear space with intact hair cells. Thus, according to the results in the gerbil clinical electrodes should be capable of reaching or even traveling within the region of functional hearing without trauma, as has recently been shown (Punte et al. 2010; Usami et al. 2011).

The present results contribute to identifying physiological markers that can be useful during an HP surgery. The CM to preserved low frequencies should be stable or increasing during a nontraumatic insertion. Reductions in the CM indicate suppression of response from intact hair cells. Small reductions are reversible so adjustment of the angle and force of insertion 
would be indicated if a deeper insertion is necessary to optimize CI hearing. Large reductions in the CM that also affect the CAP would indicate severe trauma. Such trauma is not typically reversible over the course of the acute experiments, but long-term recovery may be possible. Identifying when trauma is truly irreversible is required in future experiments to provide a reliable indication that EAS will not be possible, and the surgery should optimize CI hearing. A further desirable marker would be to indicate when the electrode is close to surviving hair cells and in an optimal position for EAS. The current results suggest that the electrode can travel nontraumatically in regions with intact hair cells, so it may be possible for the electrode to get within the zone of healthy hair cells. Whether the most apical contacts could be used or held in reserve if hearing loss is progressive is a question for future studies. Preliminary experiments in patients (personal observations) indicate that large, recordable CMs and neural responses exist in HP patients, as expected from our noise-induced hearing loss gerbil model. If it is determined that intraoperative intracochlear recording provides a reliable indication of EAS outcomes then the more detailed information seen in the gerbil recordings would clearly be of use.

\section{REFERENCES}

Adunka O, Kiefer J, Unkelbach MH, et al. Development and evaluation of an improved cochlear implant electrode design for electric acoustic stimulation. Laryngoscope. 2004; 114:1237-1241. [PubMed: 15235353]

Adunka O, Roush P, Grose J, et al. Monitoring of cochlear function during cochlear implantation. Laryngoscope. 2006; 116:1017-1020. [PubMed: 16735923]

Adunka OF, Mlot S, Suberman TA, et al. Intracochlear recordings of electrophysiological parameters indicating cochlear damage. Otol Neurotol. 2010; 31:1233-1241. [PubMed: 20818290]

Adunka OF, Pillsbury HC, Kiefer J. Combining perimodiolar electrode placement and atraumatic insertion properties in cochlear implantation-Fact or fantasy? Acta Otolaryngol. 2006; 126:475482. [PubMed: 16698696]

Büchner A, Schüssler M, Battmer RD, et al. Impact of low-frequency hearing. Audiol Neurootol. 2009; 14(suppl 1):8-13. [PubMed: 19390170]

Campbell AP, Suberman TA, Buchman CA, et al. Correlation of early auditory potentials and intracochlear electrode insertion properties: An animal model featuring near real-time monitoring. Otol Neurotol. 2010a; 31:1391-1398. [PubMed: 20856155]

Campbell AP, Suberman TA, Buchman CA, et al. Flexible cochlear microendoscopy in the gerbil. Laryngoscope. 2010b; 120:1619-1624. [PubMed: 20564668]

Cheatham MA, Naik K, Dallos P. Using the cochlear microphonic as a tool to evaluate cochlear function in mouse models of hearing. J Assoc Res Otolaryngol. 2011; 12:113-125. [PubMed: 20957507]

Choudhury B, Adunka OF, Demason CE, et al. Detection of intracochlear damage with cochlear implantation in a gerbil model of hearing loss. Otol Neurotol. 2011; 32:1370-1378. [PubMed: 21921858]

Cullen RD, Higgins C, Buss E, et al. Cochlear implantation in patients with substantial residual hearing. Laryngoscope. 2004; 114:2218-2223. [PubMed: 15564849]

Eshraghi AA, Yang NW, Balkany TJ. Comparative study of cochlear damage with three perimodiolar electrode designs. Laryngoscope. 2003; 113:415-419. [PubMed: 12616189]

Fraysse B, Macías AR, Sterkers O, et al. Residual hearing conservation and electroacoustic stimulation with the nucleus 24 contour advance cochlear implant. Otol Neurotol. 2006; 27:624-633. [PubMed: 16868510]

Gantz BJ, Hansen MR, Turner CW, et al. Hybrid 10 clinical trial: Preliminary results. Audiol Neurootol. 2009; 14(suppl 1):32-38. [PubMed: 19390173]

Gantz BJ, Turner C, Gfeller KE, et al. Preservation of hearing in cochlear implant surgery: Advantages of combined electrical and acoustical speech processing. Laryngoscope. 2005; 115:796-802.

[PubMed: 15867642] 
Gstoettner W, Kiefer J, Baumgartner WD, et al. Hearing preservation in cochlear implantation for electric acoustic stimulation. Acta Otolaryngol. 2004; 124:348-352. [PubMed: 15224851]

Gstoettner WK, van de Heyning P, O'Connor AF, et al. Electric acoustic stimulation of the auditory system: Results of a multi-centre investigation. Acta Otolaryngol. 2008; 128:968-975. [PubMed: 19086194]

Helbig S, Van de Heyning P, Kiefer J, et al. Combined electric acoustic stimulation with the PULSARCI (100) implant system using the FLEX(EAS) electrode array. Acta Otolaryngol. 2011; 131:585-595. [PubMed: 21281057]

Hochmair I, Arnold W, Nopp P, et al. Deep electrode insertion in cochlear implants: Apical morphology, electrodes and speech perception results. Acta Otolaryngol. 2003; 123:612-617. [PubMed: 12875584]

Holden, LK.; Finley, CC.; Holden, TA., et al. Factors affecting cochlear implant outcomes. Conference on Implantable Auditory Prostheses; Asilomar, CA. 2011.

Müller M. The cochlear place-frequency map of the adult and developing Mongolian gerbil. Hear Res. 1996; 94:148-156. [PubMed: 8789820]

Podskarbi-Fayette R, Pilka A, Skarzynski H. Electric stimulation complements functional residual hearing in partial deafness. Acta Otolaryngol. 2010; 130:888-896. [PubMed: 20105110]

Punte AK, Vermeire K, Van de Heyning P. Bilateral electric acoustic stimulation: A comparison of partial and deep cochlear electrode insertion. A longitudinal case study. 2010; 67:144-152.

Schmiedt RA, Zwislocki JJ. Comparison of sound-transmission and cochlear-microphonic characteristics in Mongolian gerbil and guinea pig. J Acoust Soc Am. 1977; 61:133-149. [PubMed: 833365]

Skarzynski H, Lorens A, Piotrowska A, et al. Partial deafness cochlear implantation provides benefit to a new population of individuals with hearing loss. Acta Otolaryngol. 2006; 126:934-940. [PubMed: 16864490]

Suberman TA, Campbell AP, Adunka OF, et al. A gerbil model of sloping sensorineural hearing loss. Otol Neurotol. 2011; 32:544-552. [PubMed: 21389900]

Thorne M, Salt AN, DeMott JE, et al. Cochlear fluid space dimensions for six species derived from reconstructions of three-dimensional magnetic resonance images. Laryngoscope. 1999; 109:16611668. [PubMed: 10522939]

Turner CW, Gantz BJ, Karsten S, et al. Impact of hair cell preservation in cochlear implantation: Combined electric and acoustic hearing. Otol Neurotol. 2010; 31:1227-1232. [PubMed: 20802370]

Usami S, Moteki H, Suzuki N, et al. Achievement of hearing preservation in the presence of an electrode covering the residual hearing region. Acta Otolaryngol. 2011; 131:405-412. [PubMed: 21208024]

von Ilberg C, Kiefer J, Tillein J, et al. Electric-acoustic stimulation of the auditory system. New technology for severe hearing loss. ORL J Otorhinolaryngol Relat Spec. 1999; 61:334-340. [PubMed: 10545807] 


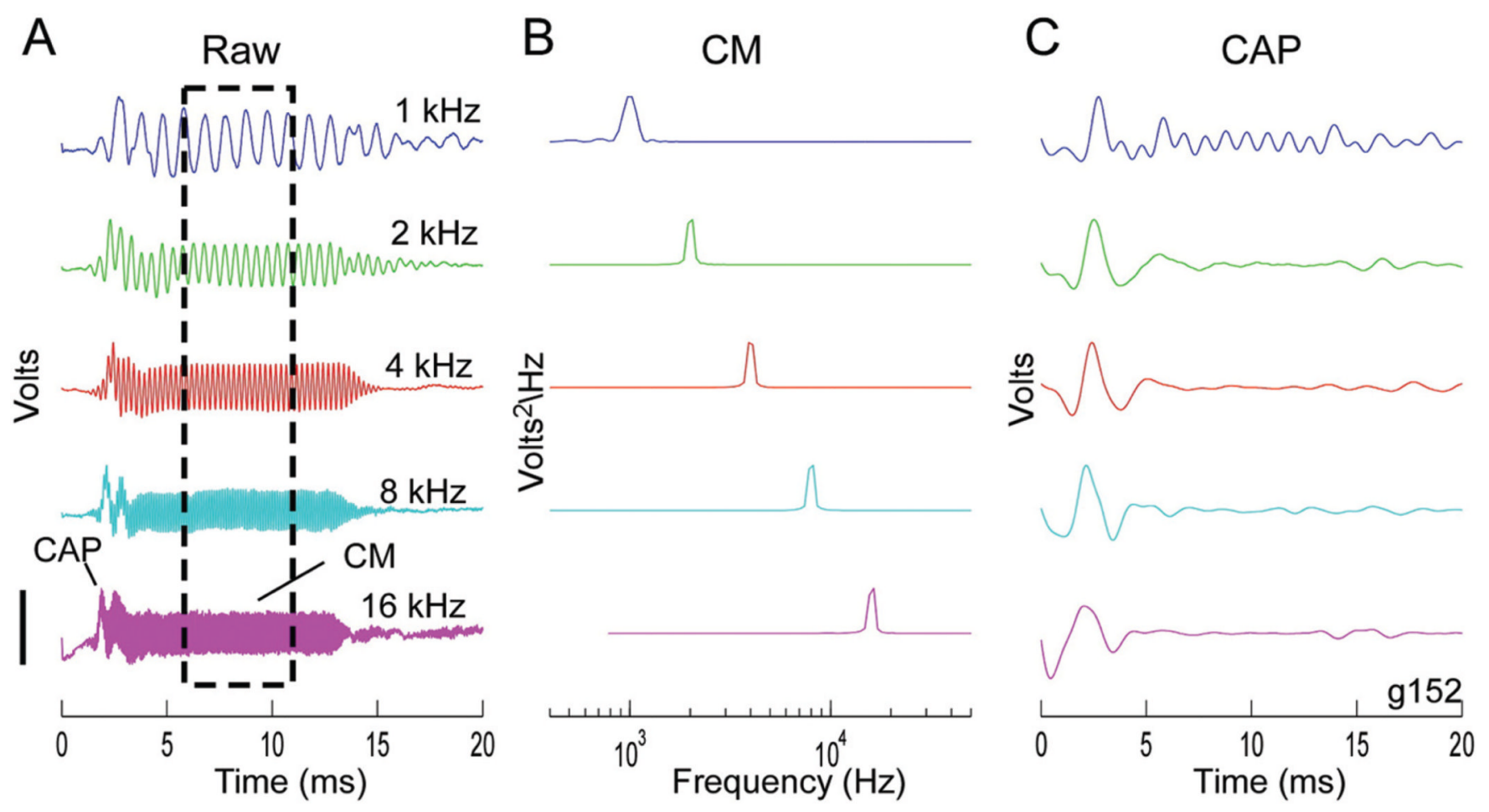

Fig. 1.

Responses to tone bursts at the round window. Sound levels were 80, 74, 53, 44, and $66 \mathrm{~dB}$ SPL for $1,2,4,8$, and $16 \mathrm{kHz}$, respectively. A, Raw signals, containing both the CM and the CAP. The box is the region of the CM used for analysis. Scale bar for the five frequencies is $116,168,195,75$, and $46 \mu \mathrm{V}$. B, CM amplitude from an FFT of the signal in the box in (A). Scale bar for the five frequencies is $98,203,378,22$, and $4 \mu \mathrm{V}^{2} / \mathrm{Hz}$. C, Signal filtered (500$1500 \mathrm{~Hz}$ ) to isolate the CAP. Scale bar for the five frequencies is $49,47,52,18$, and $12 \mu \mathrm{V}$. $\mathrm{CM}$, cochlear microphonic; CAP, compound action potential; FFT, fast-Fourier transform. 
A

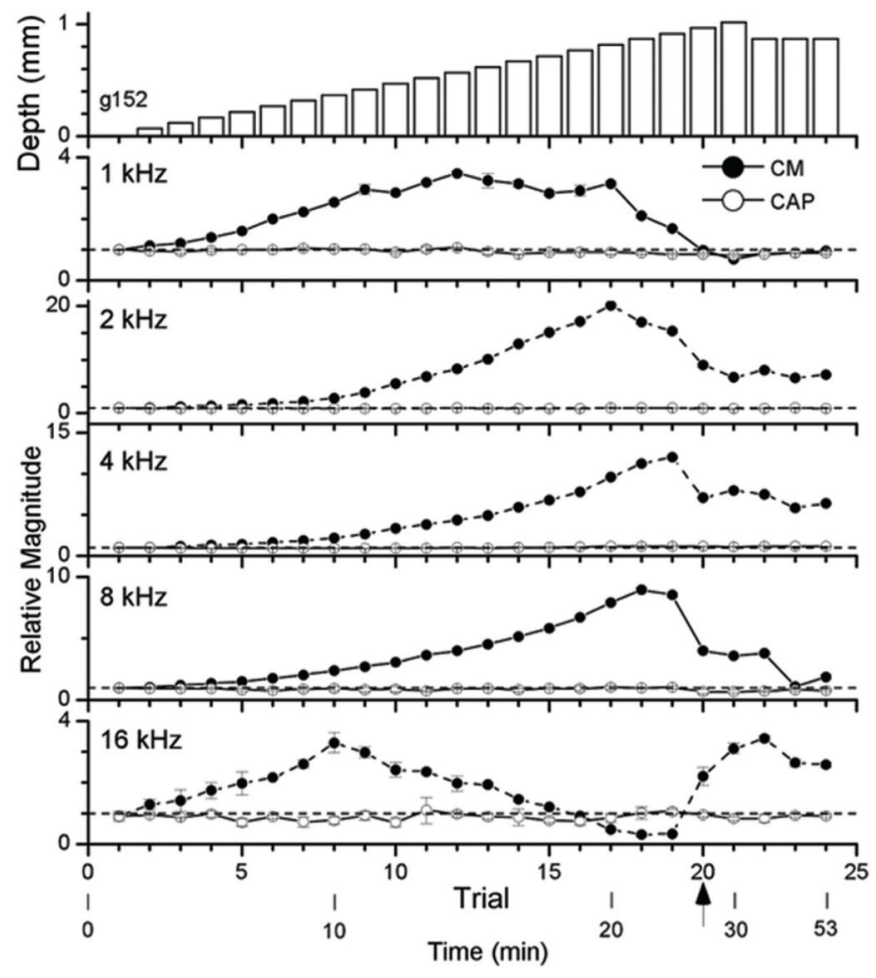

B

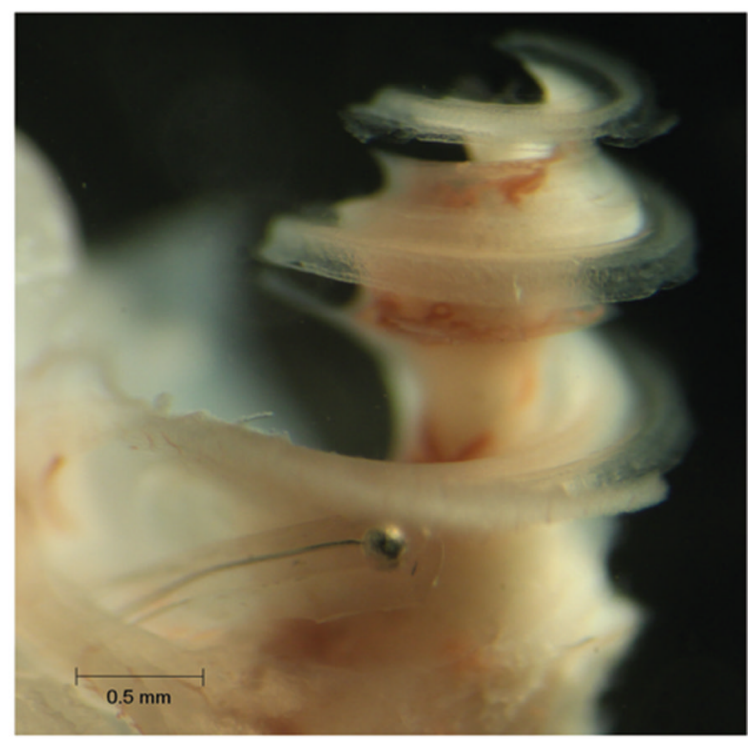

Fig. 2.

Example of an insertion of a flexible electrode through the scala tympani longitudinally. A, Physiology. The top panel is depth of insertion and the remaining panels are magnitudes of the CM (filled symbols) and the CAP (open symbols) for the different frequencies. For each of the other panels, the data points are the mean response to three blocks or 20 repetitions at each depth. The error bars, which were often smaller than the data point, are the SD. The reference value for each frequency was the magnitude of the response at the round window (dotted line), with values given in the legend of Figure 1. The CAP was relatively stable throughout but a sharp change occurred in the CM at trial 20 (arrow, $0.97 \mathrm{~mm}$ ). B, Histology. The electrode was fixed in place with wax and the end outside the cochlea was cut. The cochlea was then decalcified and viewed as a whole-mount preparation. CM, cochlear microphonic; CAP, compound action potential. 


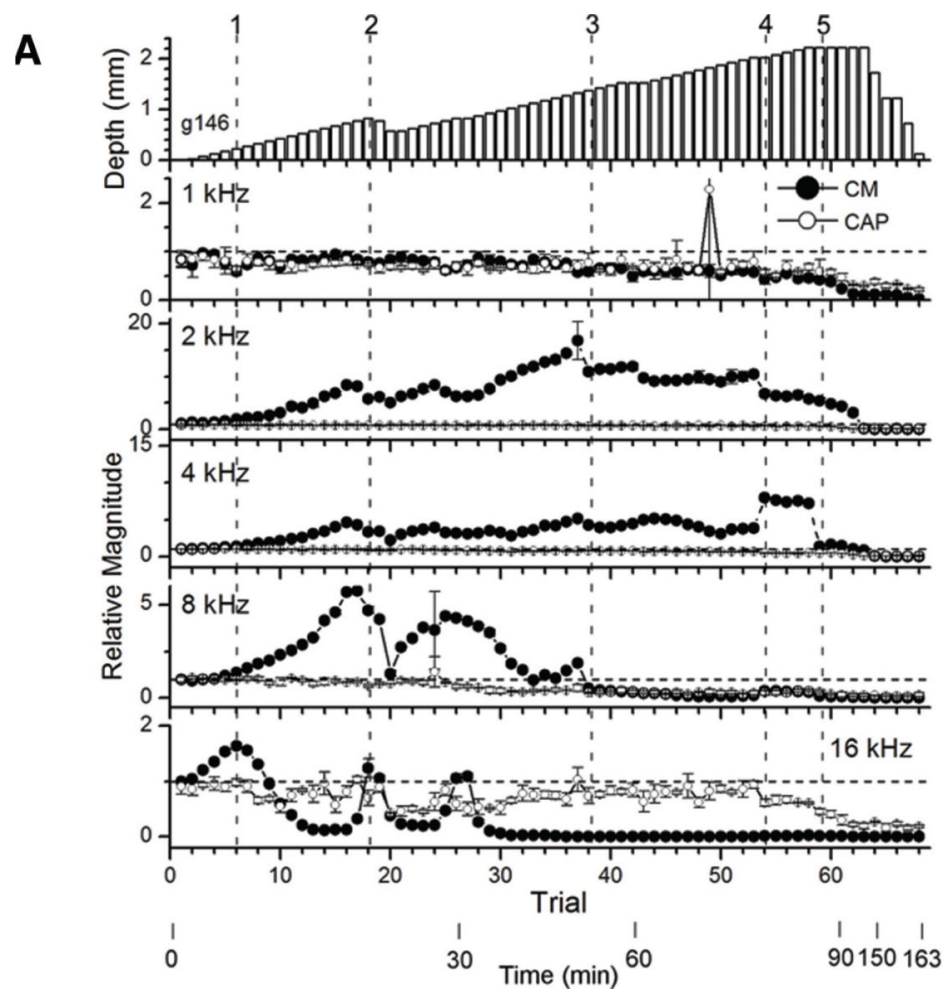

B

Fig. 3.

Example of an insertion of a flexible electrode with histological damage to the BM. A, Physiology. Format as in Figure 2. As in Figure 1, the depth in the top panel is measured from the round window, which is on average $1.34 \mathrm{~mm}$ from the hook region used to identify the site of anatomical damage. In the responses, points 1 to 5 (dotted lines) indicate transitions in the $\mathrm{CM}$ as described in the text. B, Histology. The arrow shows the hole in the RW through which the electrode was inserted, and the black arrow is the site of damage. $\mathrm{RW}$, round window; BM, basilar membrane; CM, cochlear microphonic. 


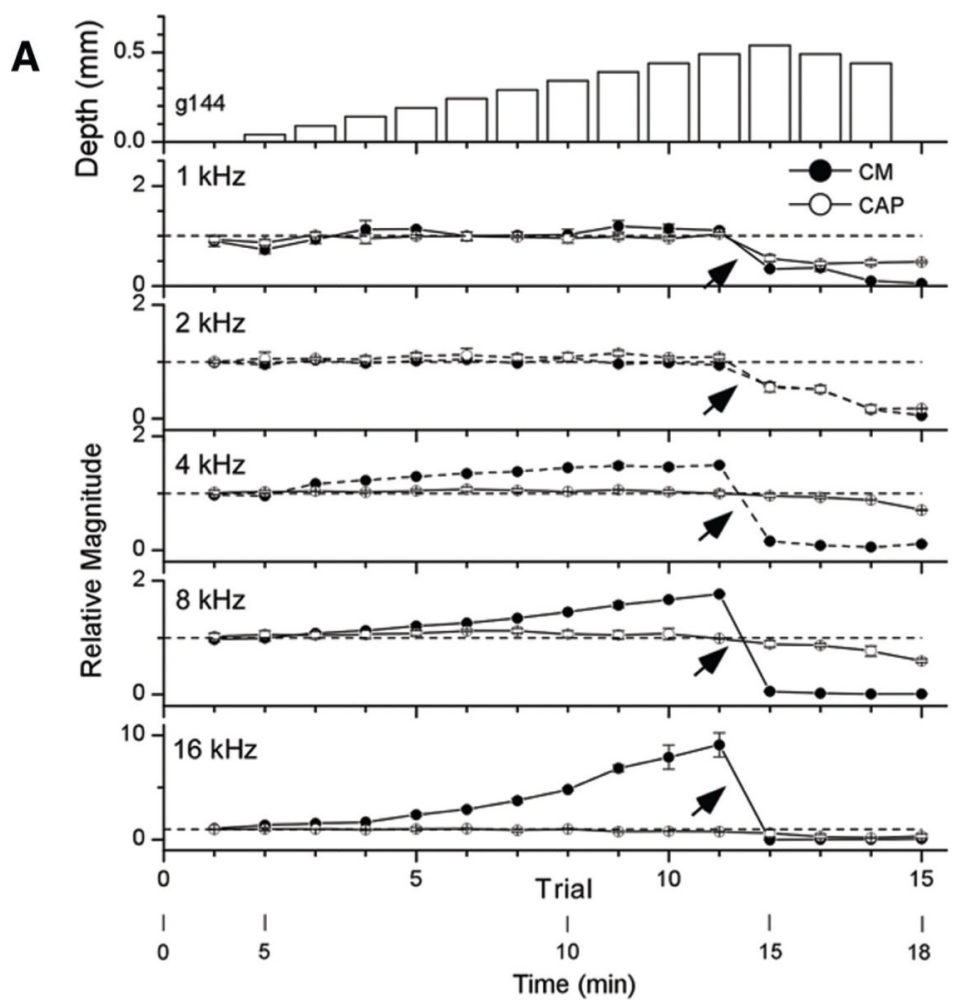

\section{B}

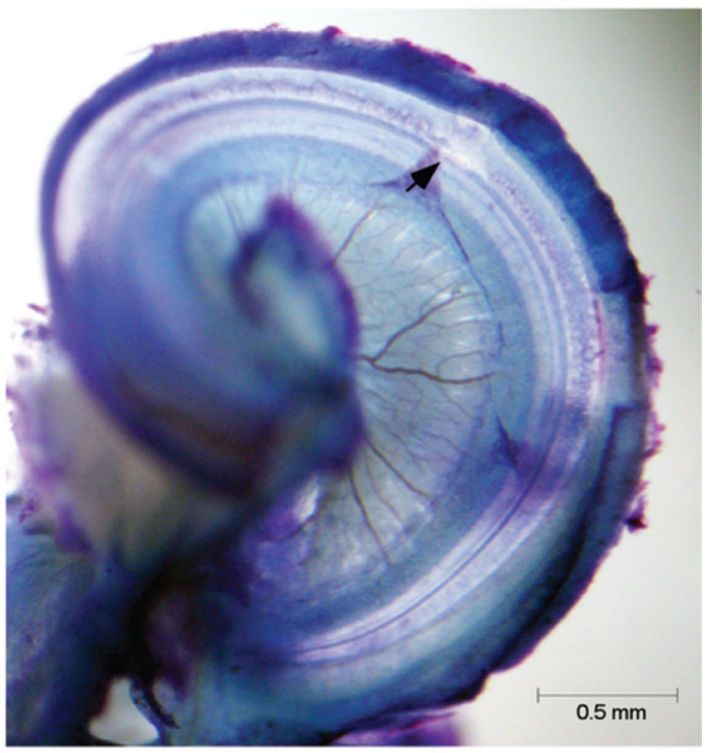

Fig. 4.

Example of an insertion of a rigid electrode with histological damage in the BM. A, Physiology. Format as in Figure 2. At trial 12 (arrows, $0.54 \mathrm{~mm}$ ) there was a sharp decline at all frequencies. B, Histology. The arrow depicts the site of damage, which was a hole in the BM. BM, basilar membrane. 


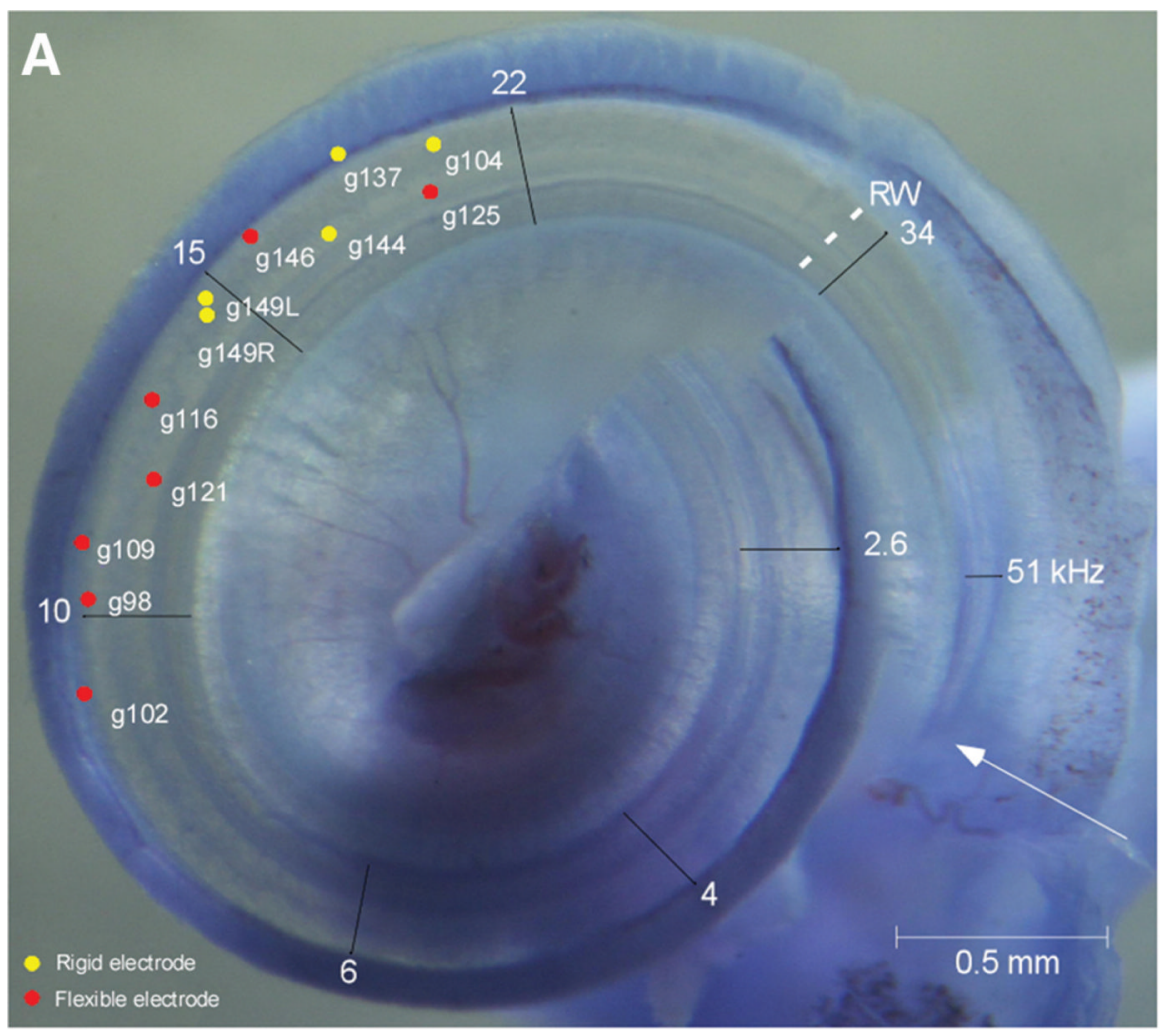

B

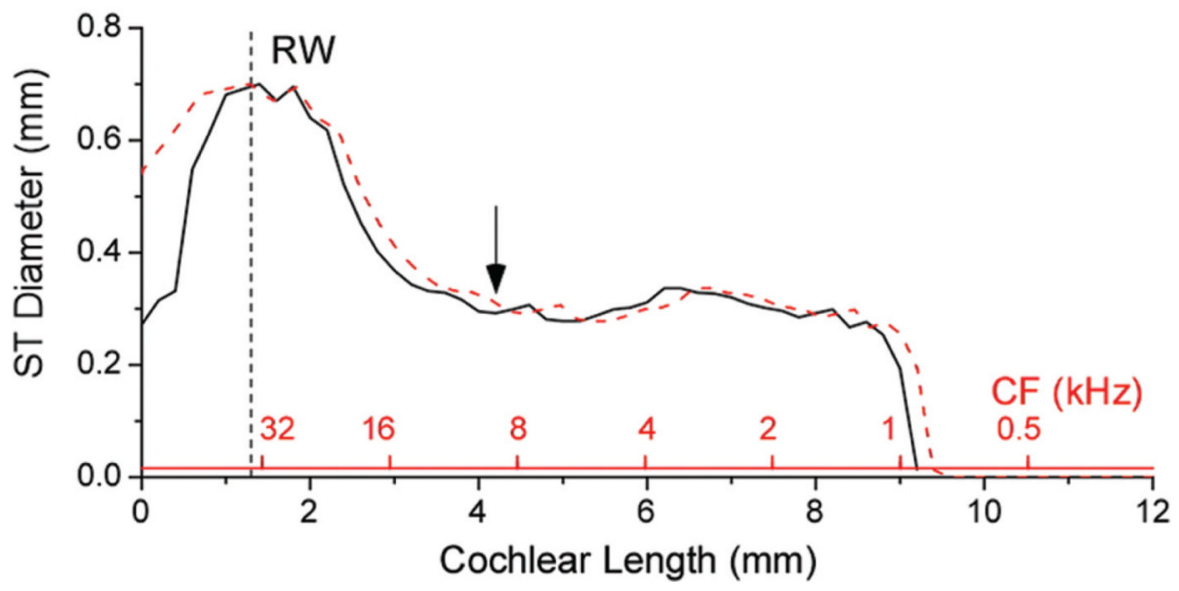

Fig. 5.

Locations of damage. A, Representative image of a cochlea whole mount, with sites of damage across cases. Frequencies are based on the gerbil place-frequency map (Muller 1996). Gerbil numbers are indicated. The deepest damage for a flexible electrode was 4.1 from the end of the hook (arrow). The deepest damage for a rigid electrode was $3.2 \mathrm{~mm}$. B, The diameter of the gerbil scala tympani as a function of cochlear distance and frequency (Thorne et al. 1999). The maximum insertion depth in (A) corresponds to the position of the arrow. RW, round window. 
